\title{
Rhodiola rosea for physical and mental fatigue: a systematic review
}

\author{
Sana Ishaque ${ }^{1}$, Larissa Shamseer ${ }^{1,2}$, Cecilia Bukutu ${ }^{3}$ and Sunita Vohra ${ }^{1 *}$
}

\begin{abstract}
Background: Rhodiola rosea (R. rosea) is grown at high altitudes and northern latitudes. Due to its purported adaptogenic properties, it has been studied for its performance-enhancing capabilities in healthy populations and its therapeutic properties in a number of clinical populations. To systematically review evidence of efficacy and safety of $R$. rosea for physical and mental fatigue.

Methods: Six electronic databases were searched to identify randomized controlled trials (RCTs) and controlled clinical trials (CCTs), evaluating efficacy and safety of $R$. rosea for physical and mental fatigue. Two reviewers independently screened the identified literature, extracted data and assessed risk of bias for included studies.

Results: Of 206 articles identified in the search, 11 met inclusion criteria for this review. Ten were described as RCTs and one as a CCT. Two of six trials examining physical fatigue in healthy populations report $R$. rosea to be effective as did three of five RCTs evaluating $R$. rosea for mental fatigue. All of the included studies exhibit either a high risk of bias or have reporting flaws that hinder assessment of their true validity (unclear risk of bias).

Conclusion: Research regarding R. rosea efficacy is contradictory. While some evidence suggests that the herb may be helpful for enhancing physical performance and alleviating mental fatigue, methodological flaws limit accurate assessment of efficacy. A rigorously-designed well reported RCT that minimizes bias is needed to determine true efficacy of $R$. rosea for fatigue.
\end{abstract}

\section{Background}

Rhodiola rosea ( $R$. rosea) is a flowering biennial grown in high latitude and altitude regions of the world. It has been a part of traditional medicine systems in parts of Europe, Asia and Russia for centuries. It has been prescribed for cancer and tuberculosis in Mongolia [1], given to newlyweds to boost fertility in Siberia [2], and used by Vikings to increase endurance and physical strength [3]. In Norway, it has even been used as food and hair wash [4].

More recently $R$. rosea has received attention from the scientific community for its potential therapeutic capacity as an adaptogen. Adaptogen are "[most commonly] natural herbal products which are non-toxic in normal doses, produce a non-specific response, and have a normalizing physiologic influence" [5]. Similarly, $R$. rosea

\footnotetext{
*Correspondence: svohra@ualberta.ca

'Complementary and Alternative Research and Education (CARE) Program, Department of Pediatrics, Faculty of Medicine and Dentistry, and School of Public Health, University of Alberta, Edmonton, Canada

Full list of author information is available at the end of the article
}

has been referred to as an ergogenic aid, i.e. an herb used to enhance physical and mental performance. Common indications pertaining to the adaptogenic and ergogenic capacity of $R$. rosea include performance enhancement, fatigue reduction and alleviation of depression symptoms. Existing reviews suggest a benefit in physical and mental performance attributable to $R$. rosea, however such reviews fail to critically appraise included literature [6,7]; some reviews rely on Russian studies that are not accessible in major international databases $[8,9]$. This systematic review aims to rigourously synthesize and appraise available clinical evidence of the efficacy of $R$. rosea for improving physical and mental performance.

\section{Active constituents}

Active constituents are biologically active components of pharmacological formulations. While it is currently unclear which specific compound(s) in $R$. rosea are active constituents, most preparations of $R$. rosea are standardized to specific levels of marker compounds rosavin, salidroside or both [8]. Rosavin is the only constituent 
unique the $R$. rosea from the Rhodiola genus; salidroside is common to most other Rhodiola species [10,11]. The naturally occurring ratio of rosavins to salidrosides in $R$. rosea is approximately $3: 1$ and $R$. rosea preparations have been prepared to reflect this ratio [12].

\section{Methods}

\section{Search strategy}

This systematic review stemmed from a larger review prepared for the Government of Alberta in 2007 for all potential indications of Rhodiola rosea. This review includes a subset of those studies examining $R$. rosea for fatigue. The term "rhodiola rosea" and common synonyms (arctic root, roseroot, rosenroot, golden root and hong jing tian) were used to search the following electronic databases: MEDLINE (1950 - July 2009), Cochrane Evidence Based Medicine databases (1991 - July 2009), EMBASE (1988 July 2009), Alternative Medicine Database (AMED) (1985 - July 2009), Natural Medicines Comprehensive database (up to July 2009) and The International Pharmaceutical Abstract Database (up to July 2009). The detailed strategy for all databases can be found in the Appendix.

\section{Inclusion criteria}

The inclusion criteria for included studies were as follows: (i) study design: any clinical trial; (ii) population: any clinical human population; (iii) intervention: Rhodiola rosea alone or in combination with other compounds; (iv) control: any comparator was considered eligible; (v) outcomes: mental or physical fatigue measured by any means. Where validated instruments were used, this information was collected.

Fatigue can be described as a pervasive sense of tiredness or lack of energy that is not related exclusively to exertion [13]. Fatigue can result due to excess physical or mental activity, sleep deprivation, and poor diet or range of medical conditions including infection, and cardiovascular, metabolic, connective tissue and endocrine disorders [13]. Division of mental and physical fatigue is arbitrary and often the two cannot be differentiated [13-15]. As such, studies describing physical or mental fatigue were both eligible for inclusion.

Due to the natural geographical distribution of the $R$. rosea plant (i.e. Scandinavian countries, Russia and parts of northern Asia), both English and non-English literature were considered. Non-English studies were translated into English.

\section{Study selection and data extraction methods}

Two authors (LS and SI) independently screened studies identified using the search strategy for inclusion, first on the basis of title and abstract and of those that were relevant, the full texts were screened for eligibility. The same two authors independently extracted data from each study using pre-developed data extraction forms. Discrepancies between reviewers were discussed and resolved by consensus. Authors of included studies were contacted to confirm and answer questions about the data.

\section{Data extraction items}

Items for which data were extracted include: publication year, country of publication, study design, population description, diagnostic criteria, age, intervention description, \% salidrosides, \% rosavins, control description, dose, duration, frequency, run-in period length, washout period length, follow-up period, number of participants randomized and analysed in each group, effect measures and measures of precision, outcome measurement tools, author's conclusions, description and number of adverse events in each group.

\section{Risk of bias assessment}

Two reviewers (LS and SI) independently assessed the risk of bias of each trial, following the domain-based evaluation endorsed by the Cochrane Collaboration [16]. The domains are as follows: randomization, concealment of allocation, blinding (of participants, personnel and outcome assessors), incomplete outcome data (whether investigators used an intention-to-treat analysis), selective outcome reporting, and other factors.

In the first three domains, and answer of "Yes" means a low risk of bias, "No" means there is a high risk of bias and "unclear" means there is an uncertain risk of bias (likely due to poor reporting). In the latter three domains, responses mean the opposite - "yes" means high risk of bias and "no" mean low risk of bias.

\section{Analysis plan}

We planned to report continuous outcomes as changes from baseline and where possible, to compare the difference between $R$. rosea and control as mean differences (MD) with $95 \%$ confidence intervals (CI). We planned to convert dichotomous outcomes into risk ratios and 95\% CIs. Meta-analysis was planned where more than one study provided data for a single outcome.

\section{Results}

\section{Description of included studies}

Two hundred and six unique articles were identified from the search and 11 met final inclusion criteria. Of those, 10 were described as randomized controlled trials (RCT) [17-26] and one was a controlled clinical trial (CCT) [24]. Six studies examined the effect of $R$. rosea on physical performance and five assessed mental fatigue. None of the studies examining $R$. rosea examining physical or mental fatigue measured outcomes consistently - no two studies reported the same outcomes. As 
such, meta-analysis could not be performed. Included studies are described in text below and Table 1.

\section{Risk of bias in trials of rhodiola rosea}

As can be seen from Figure 1, the majority of studies in each domain have an 'unclear' risk of bias in almost every domain due to how they were reported. None of the included studies are free of plausible bias (Figure 1), which raises potential concern about the validity of their results. In the domain of "other risk of bias", six studies reporting non-significant results have a low risk of bias due to lack of sample size calculation $[18,19,21,25,26]$, one calculated a sample size but did not specify a primary outcome [24] and three reported the use of outcome measurement tools that are not validated $[19,24]$.

\section{Physical fatigue}

Five RCTs and one CCT of $R$. rosea for enhancing physical performance were identified. Two trials examine a $R$. rosea-only supplement, two examined $R$. rosea-strach combined, and another two evaluate $R$. rosea combined with cordyceps.

\section{R. Rosea as single ingredient versus placebo}

A three arm double blind RCT compared the effect of $R$. rosea (as a single ingredient) to placebo, or nothing [17]. The study examined muscle recovery in 30 adults by measuring $\mathrm{C}$-reactive protein (CRP) and creatinine kinase (CK) levels in blood. Subjects underwent an exhausting physical exercise test on day 30 which consisted of cycling at $20 \mathrm{~W}$ on a bicycle ergometer with power increased by $10 \mathrm{~W} / \mathrm{min}$ until volitional exhaustion (i.e. subject could no longer pedal at $60 \mathrm{rpm}$ ). Findings indicate that $R$. rosea significantly lowered CRP levels at 5 hours and 5 days after the test $(p<0.05)$ but that CK levels were not significantly different between groups. Adverse events were not reported.

A double-blind cross-over RCT examined the effect of $R$. rosea on exercise performance in twelve male subjects [25]. Subjects received $R$. rosea or identical placebo for 3 days before outcomes were measured by an exercise test and another dose on the day of the test. A wash-out period of 7 to 14 days separated cross-over to the opposite treatment. The primary outcome was muscle recovery measured by ATP levels and secondary outcomes were time to exhaustion and perceived exertion; all outcomes were measured at baseline, during the exercise test and during recovery. There was no significant difference between groups in $\mathrm{P}_{\mathrm{i}}$, phosphocreatine and ATP levels, time to exhaustion and perceived exhaustion. Adverse events and drop-outs were not mentioned.

\section{R. Rosea plus starch versus starch alone}

One cross-over RCT and one CCT described in a single report examined the acute and long-term effects, respectively, of $R$. rosea on exercise performance [20]. In both studies, endurance capacity was the primary outcome and muscle strength, speed of limb movement, reaction time and sustained attention were secondary outcomes.

In the first study on acute effects, $R$. rosea combined with starch or placebo was taken on each of 2 days [20]. One hour after ingestion on each day, outcomes were measured while subjects underwent a physical functioning test. After a five day washout period, subjects switched to the alternate treatment and performed the same tests. Baseline measurements were not taken. Three out of six parameters of endurance capacity (time to exhaustion, $\mathrm{O}_{2}$ uptake and $\mathrm{CO}_{2}$ output) significantly improved $(p<0.05)$ in the $R$. rosea group. There was no difference between groups in any secondary outcomes. After five days, authors stated that 12 subjects were reassigned to intervention and control groups for the long-term evaluation study. The long term study evaluated subjects receiving same intervention and control as in the acute study twice per day over a four week period [20]. The same outcomes as in the acute study were measured. Long term supplementation produced no significant difference in any outcomes between treatment groups; one participant on $R$. rosea dropped out during long term supplementation for medical reasons unrelated to the study protocol (reason not stated). One subject with strong headaches during acute supplementation and one with minor headaches during long term supplementation were both on placebo. One subject experienced a minor headache and another had insomnia during long term supplementation of $R$. rosea. It is unclear why the longterm study was not randomized.

\section{R. Rosea plus cordyceps versus placebo}

Two double blind RCTs conducted evaluate the effect of $R$. rosea combined with other herbs on exercise performance $[18,21]$. Both studies were conducted by the same group of authors using slightly different protocols and populations. In both studies, intervention capsules were described as every 3 capsules containing $300 \mathrm{mg}$ of $R$. rosea (standardized to $3.0 \%$ rosavins and $2.5 \%$ salidrosidesminimum), $1000 \mathrm{mg}$ of Cordyceps sinensis, a Chinese herb reported to improve circulation [27], and $800 \mathrm{mg}$ of the manufacturers 'proprietary blend' of substances (undisclosed).

In one of the RCTs, 17 male were randomly assigned to either the $R$. rosea-containing formulation or placebo for 15 days [21]. Subjects took six capsules per day for 4 days (loading dose) then three capsules per day 11 days (maintenance dose). Endurance capacity was measured 
Table 1 Rhodiola rosea summary of clinical evidence PHYSICAL PERFORMANCE

\begin{tabular}{|c|c|c|c|c|}
\hline \multicolumn{5}{|c|}{ R. rosea single ingredient versus placebo } \\
\hline Study ID & Design & Population & Intervention/Control & Outcome(s) \\
\hline $\begin{array}{l}\text { Abidov } \\
2004\end{array}$ & $\mathrm{DB} R C T$ & $\begin{array}{l}36 \text { male and female non-smokers } \\
\text { between } 21-24 \text { y.o.a. }\end{array}$ & $\begin{array}{l}\text { Int: } 340 \text { mg RR extract containing } \\
30 \text { mg active RR (including rosavin) }\end{array}$ & $\begin{array}{l}\text { CRP blood-levels } 5 \mathrm{~h} \text { and } 5 \text { days after } \\
\text { exercise were less than placebo and }\end{array}$ \\
\hline \multirow[t]{3}{*}{ (Russia) } & & & Con(1): placebo & \\
\hline & & & Con(2): no intervention; & \multirow{2}{*}{$\begin{array}{c}\text { CK levels in blood same across groups } \\
\text { after } 5 \text { hours, but reduced after } 5 \text { days in } \\
\text { only } R \text {. rosea group. }\end{array}$} \\
\hline & & & $\begin{array}{l}\text { Regimen: for } 30 \text { days before and } 6 \text { days } \\
\text { after exhausting physical exercise. }\end{array}$ & \\
\hline \multirow[t]{2}{*}{$\begin{array}{l}\text { Walker } \\
2007 \\
\text { (US) }\end{array}$} & DB CO RCT & $\begin{array}{l}12 \text { resistance-trained males } \\
(19-39 \text { y.o.a. })\end{array}$ & $\begin{array}{l}\text { Int: } 1500 \text { mg/day RR for } 3 \text { days before } \\
\text { exercise test + } 1000 \text { mg on day of test } \\
\text { Con: placebo; }\end{array}$ & \multirow[t]{2}{*}{$\begin{array}{c}\text { No significant differences between } \\
\text { groups in measures of ATP kinetics and } \\
\text { exhaustion. }\end{array}$} \\
\hline & & & $\begin{array}{l}\text { Regimen: } \mathrm{RR} \text { followed by } 7-14 \text { days } \\
\text { washout and same dosing regimen of } \\
\text { placebo OR treatment in reverse order }\end{array}$ & \\
\hline
\end{tabular}

\section{R. rosea Plus starch versus placebo}

\begin{tabular}{|c|c|c|c|c|}
\hline $\begin{array}{l}\text { De Bock } \\
2004 a\end{array}$ & DB CO RCT & $\begin{array}{l}24 \text { healthy physically active male } \\
(21.8 \pm 0.3 \text { y.o.a) and female }\end{array}$ & Int:100 mg RR +250 mg starch & $\begin{array}{l}\text { RR 'significantly' delayed time to } \\
\text { exhaustion, peak } \mathrm{O}_{2} \text { uptake and } \mathrm{CO}_{2}\end{array}$ \\
\hline \multirow[t]{2}{*}{ (Belgium) } & & & Con: 350 mg starch & \\
\hline & & & $\begin{array}{c}\text { Regimen: } 2 \text { days of RR, } 5 \text { days washout, } \\
2 \text { days placebo }\end{array}$ & \\
\hline $\begin{array}{l}\text { De Bock } \\
2004 b\end{array}$ & DB CCT & & Int:100 mg RR +250 mg starch & $\begin{array}{c}\text { significantly higher blood lactate levels } \\
\text { after 4-wk intake. }\end{array}$ \\
\hline \multirow[t]{2}{*}{ (Belgium) } & \multicolumn{4}{|c|}{ Con: 350 mg starch } \\
\hline & & & $\begin{array}{c}\text { Regimen: RR or placebo twice/day over } \\
\qquad 4 \text { weeks }\end{array}$ & $\begin{array}{c}\text { Overall, no change in muscle strength, } \\
\text { speed of limb movement, reaction time, } \\
\text { sustained attention. }\end{array}$ \\
\hline \multicolumn{5}{|c|}{ R. rosea plus Cordyceps versus Placebo } \\
\hline \multirow{4}{*}{$\begin{array}{l}\text { Earnest } \\
2004 \text { (US) }\end{array}$} & \multirow[t]{4}{*}{ DB RCT } & 17 male competitive cyclists & \multirow{3}{*}{$\begin{array}{l}\text { Int: loading dose of } 6 \text { capsules/day for } \\
4 \text { days (every three capsules contain } \\
1000 \text { mg Cordyceps sinensis }+300 \text { mg RR) } \\
\text { then maintenance dose of } 3 \text { capusles/ } \\
\text { day for } 11 \text { days }\end{array}$} & \multirow{4}{*}{$\begin{array}{l}\text { No significant difference between or } \\
\text { within Tx groups in peak and subpeak } \\
\text { exercise variables. }\end{array}$} \\
\hline & & int: $31.6 \pm 2.8$ (SE) y.o.a. & & \\
\hline & & con: $30.5 \pm 2.2$ y.o.a. & & \\
\hline & & & Con: identical placebo & \\
\hline \multirow[t]{2}{*}{$\begin{array}{l}\text { Colson } \\
2005 \text { (US) }\end{array}$} & \multirow[t]{2}{*}{ DB RCT } & 8 males between $18-34$ y.o.a. & $\begin{array}{l}\text { Int: loading dose of } 6 \text { capsules/day for } \\
6 \text { days (every three capsules contain } \\
1000 \text { mg Cordyceps sinensis }+300 \text { mg RR) } \\
\text { then maintenance dose of } 3 \text { capsules/ } \\
\text { day for } 7 \text { days }\end{array}$ & \multirow[t]{2}{*}{$\begin{array}{l}\text { After pre-post endurance test no } \\
\text { significant difference between } \\
\text { intervention and placebo in muscle } \\
\text { tissue oxygen saturation }\end{array}$} \\
\hline & & & Con: identical placebo & \\
\hline \multicolumn{5}{|c|}{ MENTAL PERFORMANCE } \\
\hline Study ID & Design & Population & Intervention/Control & Outcome(s) \\
\hline \multirow[t]{3}{*}{$\begin{array}{l}\text { Olsson } \\
2008\end{array}$} & \multirow[t]{3}{*}{ DB RCT } & $\begin{array}{l}60 \text { male and female ( } 20-55 \text { y.o.a) } \\
\text { with fatigue syndrome }\end{array}$ & $\begin{array}{c}\text { Int: } 4 \text { Verum tablets/day for } 28 \text { days } \\
\text { (each tablet contain } 144 \text { mg Rhodiola } \\
\text { extract SHR-5) }\end{array}$ & \multirow{3}{*}{$\begin{array}{l}\text { Significant improvement in fatigue } \\
\text { scores (measured by Pines burnout } \\
\text { scale), and significant improvement in } \\
\text { two of five CCPT II indices }\end{array}$} \\
\hline & & int: $41.0 \pm 7.9$ y.o.a & \multirow[t]{2}{*}{ Con: identical placebo } & \\
\hline & & con: $42.1 \pm 8.5$ y.o.a. & & \\
\hline $\begin{array}{l}\text { Darbinyan } \\
2000\end{array}$ & DB CO RCT & $\begin{array}{l}56 \text { male and female physicians } \\
\text { on night duty }\end{array}$ & $\begin{array}{l}\text { Group A: Standardized extract of } 170 \mathrm{mg} \\
\text { RR for } 2 \text { weeks; } 2 \text { week Washout; }\end{array}$ & $\begin{array}{c}\text { Significant improvement in total fatigue } \\
\text { score after two weeks on RR; }\end{array}$ \\
\hline \multirow[t]{2}{*}{ (Armenia) } & & Group A: $25.5 \pm 3.8$ y.o.a. & \multirow{2}{*}{$\begin{array}{c}2 \text { weeks identical placebo. Group B: } \\
\text { treatment in reverse order }\end{array}$} & \\
\hline & & Group B: $27.3 \pm 2.9$ y.o.a. & & \\
\hline $\begin{array}{l}\text { Shevstov } \\
2003\end{array}$ & DB RCT & $\begin{array}{l}121 \text { male military cadets } 19-21 \\
\text { y.o.a. }\end{array}$ & $\begin{array}{c}\text { Int: } 41 \text { subjects }-2 \text { capsules }(185 \mathrm{mg} \\
\text { each) RR. }\end{array}$ & \multirow{2}{*}{$\begin{array}{c}\text { Total antifatigue index scores } \\
\text { significantly lower in both RR groups } \\
\text { than placebo }(p<0.0001) \text {. }\end{array}$} \\
\hline (Russia) & & & 20 subjects -3 capsules RR & \\
\hline
\end{tabular}


Table 1 Rhodiola rosea summary of clinical evidence (Continued)

\begin{tabular}{|c|c|c|c|c|}
\hline & & & Con: 40 subjects - identical placebo & "statistically significant beneficial \\
\hline & & & 20 subjects - untreated control & $\begin{array}{c}\text { versus the placebo group" } \\
\text { ves }\end{array}$ \\
\hline \multirow{2}{*}{$\begin{array}{l}\text { Spasov } \\
2000 \\
\text { (Russia) }\end{array}$} & \multirow{2}{*}{$\begin{array}{l}\text { DB RCT } \\
\text { (pilot } \\
\text { study) }\end{array}$} & \multirow[t]{2}{*}{$\begin{array}{l}40 \text { Male students from India } \\
17-19 \text { y.o.a. }\end{array}$} & $\begin{array}{c}\text { Int: } 2 \times 50 \text { mg RR tablets twice/day for } \\
20 \text { days }\end{array}$ & \multirow{2}{*}{$\begin{array}{l}\text { Non-significant improvement in physica } \\
\text { work and mental capacity. Significant } \\
\text { improvement in general well-being } \\
(p<0.05) \text {, mental fatigue }(p<0.01) \text {. }\end{array}$} \\
\hline & & & Con: identical placebo & \\
\hline $\begin{array}{l}\text { Spasov } \\
2000 \\
\text { translated } \\
\text { (Russia) }\end{array}$ & RCT & $\begin{array}{c}60 \text { male students from India } \\
(17-18 \text { y.o.a.) }\end{array}$ & $\begin{array}{c}\text { Int: } 660 \text { mg/day for } 20 \text { days RR; Con(1): } \\
\text { placebo; Con(2): nothing }\end{array}$ & $\begin{array}{c}\text { Measures of subjective self-evaluation, } \\
\text { psychological fatigue, situational anxiety, } \\
\text { motivation, precision of motor function, } \\
\text { process of excitement and need for rest } \\
\text { are significantly different within RR } \\
\text { group }(<0.05) \text {. Mean changes in mental } \\
\text { work capacity and neuromotor function } \\
\text { are not significantly different between } \\
\text { groups. }\end{array}$ \\
\hline
\end{tabular}

Abbreviations: RR Rhodiola rosea, DB double-blind, RCT Randomized controlled trial, CCT Controlled clinical trial, CO cross-over, Int Intervention, Con Control, DO Drop-outs, $A E$ Adverse events.

by multiple parameters including peak $\mathrm{CO}_{2}$ output, power output, time to exhaustion and peak heart rate, which were measured at the beginning and end of the study period. The authors found that the herbal formulation did not have any significant effect on exercise endurance or capacity. Adverse events were not reported.

The second study involving eight male cyclists randomized to either $R$. rosea-containing formulation $(33.0 \pm 12.6)$ years) or placebo $(23.8 \pm 2.9$ years $)$, followed the same protocol as above, however the study period was only 13 days - 6 days of the loading dose and 7 days of the maintenance dose [18]. Respiratory parameters were measured in the participants. This study also found no significant difference in outcomes between groups. There were no drop outs; adverse events were not mentioned.

\section{Mental fatigue}

A double blinded RCT assessed the efficacy of a $R$. rosea extract, SHR-5, for stress related fatigue [22]. Sixty subjects were randomized to receive $576 \mathrm{mg}$ of $R$. rosea preparation or placebo per day for 28 days. Mental fatigue, measured by the Pines burnout scale, was the primary outcome. Other outcomes evaluated were depression (Montgomery-Asberg Depression Rating Scale, MADRS), quality of life (Medical Outcomes Study Short form 36-item questionnaire, SF-36), attention (Conners' Computerized Continuous Performance Test II, CCCPT II) and "anti-fatigue" effect (saliva cortisol response after awakening). All outcomes were measured before and after the treatment period. The Pines burnout scale scores $(p=0.047)$ and two out of five indices of CCCPT II $(p=0.02, p=0.001)$ improved in favour or $R$. rosea. While investigators conclude that the treatment appears to have beneficial effect, they report excluding follow-up data for at least 5 participants due to physical loss of data and protocol deviations. Per-protocol analyses (i.e. analysis of only participants who followed the protocol for the entirety of the study) may overestimate treatment effect if the reasons for incomplete data are related to the treatment effect $[28,29]$ - in this case, it is not explicitly stated what "protocol deviations" occurred. No adverse effects occurred during the study period.

$R$. rosea for non-specific fatigue was evaluated in a double-blind crossover RCT in 56 Armenian physicians [19]. Participants were randomized to either $170 \mathrm{mg} R$. rosea (standardized to $2.6 \%$ salidroside) or placebo. The study period lasted for two weeks followed by a twoweek wash-out period, after which participants were crossed over for two weeks. The primary outcome was fatigue, measured using a fatigue index developed for use in this study; the tool does not appear to be validated. Measurements were carried out before and after the treatment period. Authors state that they found a significant improvement in the fatigue index after two weeks of $R$. rosea supplementation, but only present data for the 5 individual test scores. Since we are unable to replicate and confirm their analysis, findings of this study must be interpreted as inconclusive. Authors indicate that no adverse events occurred; whether or not anyone dropped out of the study was not reported.

A double-blinded RCT conducted in Russia evaluated the effect of two different single doses of $R$. rosea on mental fatigue [23]. Subjects were randomized to take $R$. rosea or placebo. A non-treatment group was also included, however subjects were not randomized into this group and comparisons against this group will not be considered in this review. The intervention was taken at 4:00 am while participants were on an overnight shift. Capacity for mental work, measured using a fatigue index of unknown origins and pulse pressure and rate were evaluated before night duty and one hour after taking the study medication. A self-report questionnaire evaluating general well-being was completed after taking the study medication. The fatigue index was comprised 


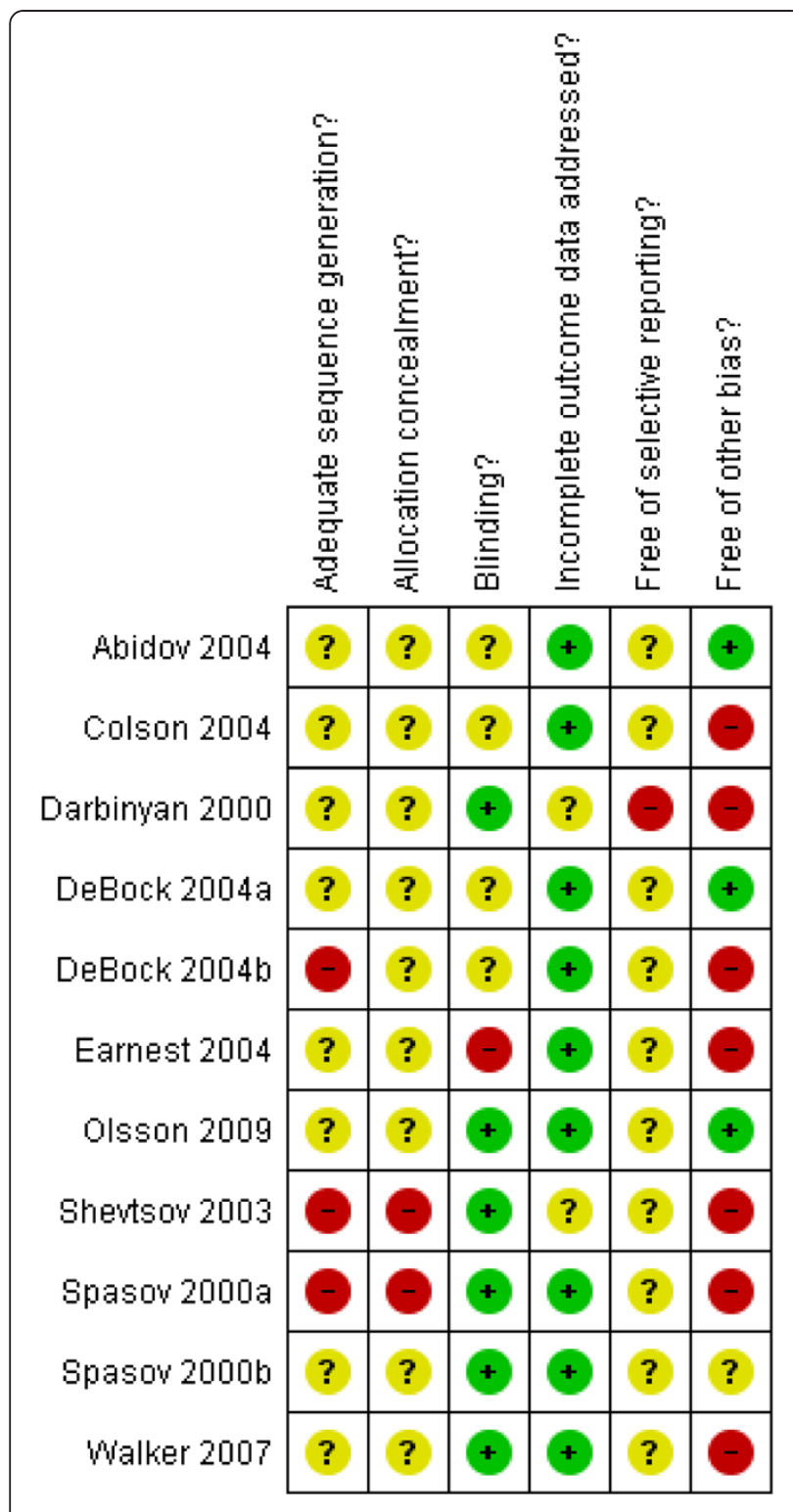

Figure 1 Risk of bias assessment in included studies.

of three parameters: visual perception, short-term memory and perception of order. Improvements in favour of both doses of $R$. rosea were apparent in the fatigue index $(p<0.001)$; no significant differences between groups occurred for other outcomes. The method of randomization was unclear. One subject in the placebo group experienced hypersalivation; whether or drop-outs occurred was not reported.

A double-blinded RCT pilot study examined the effect of a repeated low dose of $R$. rosea on foreign students' mental and physical well-being during their examination period [24]. Subjects were randomized into 2 groups to receive either $100 \mathrm{mg} R$. rosea once per day or identical placebo for 20 days. Hand-eye coordination (maze test), motoric speed (tapping test), mental work capacity (correction of text test), fatigue and well-being (self-evaluation questionnaire), heart rate and physical work capacity (bicycle ergometer test) were assessed. Significant improvements were observed in hand-eye coordination $(p<0.01)$, mental fatigue and general-well being $(p<0.01)$ in favour of $R$. rosea. Students on placebo had a significantly higher heart rate $(p<0.05)$. Drop-outs and adverse events were not reported by authors.

Another RCT conducted by the same group examined 60 male students in their first year of study at a Russian high school [26]. Students were randomized into 3 groups to receive either of Rhodaxon $(R$. rosea extract with no ethyl alcohol per day; proportions of active constituents not given), placebo or nothing for 20 days. Participants underwent the same tests for mental and physical capacity as above as well as a psychophysiological test [Lusher test [30]] to determine level of anxiety, psychological fatigue and need to rest. A comparative analysis between groups was not conducted leaving the effect of $R$. rosea indeterminable. Adverse events and drop-outs were not reported.

\section{Adverse effects}

Out of 446 subjects examined in the 11 included clinical studies, five adverse events were mentioned in three studies. Two subjects on $200 \mathrm{mg}$ of $R$. rosea over a 4week period each experienced a minor and serious headache [24]; one subject on placebo over a 2-day treatment period experienced a minor headache and another had insomnia [20]. Another subject on placebo experienced hypersalivation [23]. There appear to be few side effects associated with $R$. rosea supplementation; those identified are of a mild nature.

\section{Discussion}

Even though 10 of the 11 included studies are RCTs, there is insufficient evidence to evaluate the effectiveness of Rhodiola rosea on physical or mental fatigue. While RCTs are the modern day gold standard for assessment of efficacy of medical interventions, none of the included RCTs appeared to be compliant with the CONSORT (Consolidated Standards of Reporting Trials) Statement, an internationally agreed-upon set of RCT reporting guidelines [31]. In addition, appraisal using the Cochrane Collaboration's new tool for assessing bias in RCTs, indicates a largely unclear or high risk of bias within included studies.

Of the five RCTs of $R$. rosea for enhancing mental performance identified, three indicated that the herb may be effective in improving overall health in a mentally fatigued population. However, two of these studies did not appear to use validated measures of fatigue, making 
the validity of their findings difficult to assess. The remaining studies offered inconclusive or negative results. Further rigorous RCTs are required in order to determine the effect of $R$. rosea on mental fatigue.

$R$. rosea has demonstrated a very low occurrence of side effects demonstrating a low clinical toxicity. Although no contraindications with other herbal or prescription medications have been identified, it is important to consider that $R$. rosea may have an additive effect with other substances exhibiting stimulant properties [32]. Like many natural health products, the likelihood of adequate reporting of adverse events may be lower than conventional medications [33].

Clinical studies report $R$. rosea-only products ranging in dose from $50 \mathrm{mg}$ to $660 \mathrm{mg}$ per capsule, to a maximum of $1500 \mathrm{mg} /$ day, suggesting a large margin of safety. Studies reporting a positive effect of $R$. rosea on physical performance reported doses of $200 \mathrm{mg} /$ day and $680 \mathrm{mg} /$ day and those reporting a positive effect on mental fatigue reported doses between 100-576 mg/day.

\section{Limitations}

It is not possible to know the sole effect of $R$. rosea from combination interventions. However our goal was to be comprehensive in our approach, and these studies were included in case they provided interesting hypothesis generating questions for future research.

No studies in this review specifically included pediatric populations or pregnant or lactating women. As well, pregnant or lactating women were explicitly excluded from the majority of clinical studies. No indications have been identified which are specifically relevant to either of these populations, nor have reports of toxicity or adverse events. Overall, due to the paucity of clinical studies including these populations, no dosage recommendations can be made until further studies have been conducted which evaluate the safety and/or toxicity of $R$. rosea in children and pregnant or lactating women.

\section{Conclusion}

The current evidence for efficacy of $R$. rosea is contradictory and inconclusive. Methodologically rigorous RCTs must be designed to overcome these serious threats to internal validity. Such studies will help inform policymakers, health care providers, and the public about the efficacy $R$. rosea supplementation for physical and mental performance.

\section{Appendix}

\section{Medline search}

1. rhodiola rosea.mp. [mp $=$ title, original title, abstract, name of substance word, subject heading word]

2. roseroot.mp. $[\mathrm{mp}=$ title, original title, abstract, name of substance word, subject heading word]
3. golden root.mp. $[\mathrm{mp}=$ title, original title, abstract, name of substance word, subject heading word]

4. arctic root.mp. [mp = title, original title, abstract, name of substance word, subject heading word]

5. king's crown.mp. [mp = title, original title, abstract, name of substance word, subject heading word]

6. rosenroot.mp. $[\mathrm{mp}=$ title, original title, abstract, name of substance word, subject heading word]

7. rodia riza.mp. $[\mathrm{mp}=$ title, original title, abstract, name of substance word, subject heading word]

8. lignum rhodium.mp. [mp $=$ title, original title, abstract, name of substance word, subject heading word]

9. sedum rhodiola.mp. $[\mathrm{mp}=$ title, original title, $\mathrm{ab}$ stract, name of substance word, subject heading word]

10. sedum rosea.mp. $[\mathrm{mp}=$ title, original title, abstract, name of substance word, subject heading word]

11. hong jing tian.mp. $[\mathrm{mp}=$ title, original title, abstract, name of substance word, subject heading word]

12. exp Rhodiola/

13. or/1-12

14. limit 13 to humans

\section{Cochrane database of systematic reviews (the whole} suite)

1. Rhodiola/

2. (roseroot or golden root or arctic root).mp. [mp = title, original title, abstract, mesh headings, heading words, keyword]

3. (king's crown or rosenroot or rodia riza or lignum rodium or lignum rhodium or sedum rhodiola or sedum rosea or hong jing tian).mp. [mp = title, original title, abstract, mesh headings, heading words, keyword]

4. 1 or 2 or 3

5. humans/

6. 4 and 5

\section{Amed}

1. exp Rhodiola/

2. rhodiola rosea.mp.

3. roseroot.mp.

4. golden root.mp.

5. arctic root.mp. [mp = abstract, heading words, title]

6. king's crown.mp. $[\mathrm{mp}=$ abstract, heading words, title]

7. rhodiola.mp. [mp = abstract, heading words, title]

8. rosenroot.mp. $[\mathrm{mp}=$ abstract, heading words, title]

9. rodia riza.mp. $[\mathrm{mp}=$ abstract, heading words, title]

10. lignum rhodium.mp. $[\mathrm{mp}=$ abstract, heading words, title]

11. sedum rhodiola.mp. [ $\mathrm{mp}=$ abstract, heading words, title]

12. sedum rosea.mp. $[\mathrm{mp}=$ abstract, heading words, title] 
13. hong jing tian.mp. [ $\mathrm{mp}=$ abstract, heading words, title]

14. 4 or 1 or 3 or 7 or 12 or 2IPA

1. rhodiola.mp. $[\mathrm{mp}=$ title, subject heading word, registry word, abstract, trade name/generic name]

2. roseroot.mp. $[\mathrm{mp}=$ title, subject heading word, registry word, abstract, trade name/generic name]

3. golden root.mp. [mp = title, subject heading word, registry word, abstract, trade name/generic name]

4. arctic root.mp. [mp = title, subject heading word, registry word, abstract, trade name/generic name]

5. king's crown.mp. [mp = title, subject heading word, registry word, abstract, trade name/generic name]

6. rosenroot.mp. $[\mathrm{mp}=$ title, subject heading word, registry word, abstract, trade name/generic name]

7. rosen root.mp. [mp = title, subject heading word, registry word, abstract, trade name/generic name]

8. rodia riza.mp. $[\mathrm{mp}=$ title, subject heading word, registry word, abstract, trade name/generic name]

9. lignum rhodium.mp. [mp = title, subject heading word, registry word, abstract, trade name/generic name]

10. sedum rhodiola.mp. [mp = title, subject heading word, registry word, abstract, trade name/generic name]

11. sedum rosea.mp. [mp = title, subject heading word, registry word, abstract, trade name/generic name]

12. hong jing tian.mp. $[\mathrm{mp}=$ title, subject heading word, registry word, abstract, trade name/generic name]

13. or/1-12

14. limit 13 to human

\section{Embase}

1. exp Rhodiola Extract/or exp Rhodiola/or exp Rhodiola Rosea Extract/

2. exp rhodiola rosea/or rhodiola rosea.mp.

3. roseroot.mp.

4. golden root.mp.

5. arctic root.mp.

6. king's crown.mp.

7. rosenroot.mp. [mp = title, abstract, subject headings, heading word, drug trade name, original title, device manufacturer, drug manufacturer name]

8. rosen root.mp. $[\mathrm{mp}=$ title, abstract, subject headings, heading word, drug trade name, original title, device manufacturer, drug manufacturer name]

9. rodia riza.mp. [ $\mathrm{mp}=$ title, abstract, subject headings, heading word, drug trade name, original title, device manufacturer, drug manufacturer name]

10. lignum rhodium.mp. [mp = title, abstract, subject headings, heading word, drug trade name, original title, device manufacturer, drug manufacturer name]

11. sedum rhodiola.mp. $[\mathrm{mp}=$ title, abstract, subject headings, heading word, drug trade name, original title, device manufacturer, drug manufacturer name]
12. sedum rosea.mp. [ $\mathrm{mp}=$ title, abstract, subject headings, heading word, drug trade name, original title, device manufacturer, drug manufacturer name]

13. hong jing tian.mp. $[\mathrm{mp}=$ title, abstract, subject headings, heading word, drug trade name, original title, device manufacturer, drug manufacturer name]

14. or/1-13

15. limit 14 to human

\section{Competing interest}

SV receives salary support from Alberta Innovates-Health Solutions. None of the authors have a personal or financial competing interest.

\section{Authors' contributions}

All authors read and approved the final manuscript. Author contributions were as follows: SV conceived of the review, LS, CB and SV designed the review, LS, CB and SI conducted the review (performed screening, data extraction, risk of bias assessment); LS analysed dated; SI, LS and CB contributed to the writing of the review; SV provided guidance and supervision and has primary responsibility for final content. All authors critically revised the manuscript for important intellectual content.

\section{Financial disclosure}

This project was funded by Canadian Foundation for Innovation (New Initiatives fund 2004/2005), Alberta Value-Added Corporation (AVAC), Alberta Crop Industry Development Fund (ACIDF), Alberta Agriculture and Rural Development (AARD). Funders did not play any role in the concept and design of the study.

\section{Acknowledgment}

The authors would like to thank Soleil Surette for help refining and performing the search.

\section{Author details}

${ }^{1}$ Complementary and Alternative Research and Education (CARE) Program, Department of Pediatrics, Faculty of Medicine and Dentistry, and School of Public Health, University of Alberta, Edmonton, Canada. ${ }^{2}$ Clinical Epidemiology Program, Ottawa Hospital Research Institute, Ottawa, Canada.

${ }^{3}$ Alberta Centre for Child, Family \& Community Research, Edmonton, Canada.

Received: 12 August 2011 Accepted: 10 April 2012

Published: 29 May 2012

\section{References}

1. Khaidaev Z, Menshikova TA: Medicinal plants in Mongolian medicine. Mongolia: Ulan-Bator; 1978.

2. Saratikov SA, Krasnov EA: Rhodiola rosea is a valuable medicinal plant (Golden Root). Tomsk, Russia: Tomsk State University Press; 1987.

3. Magnusson B: Beauty: herbs that touch us. Ostersun, Sweden: Berndtssons; 1992:66-67.

4. Alm T: Ethnobotany of Rhodiola rosea (Crassulaceae) in Norway. SIDA 2004, 21(1):321-344.

5. Brekhman I, Dardymov IV: New substances of plant origin which increase non-specific resistance. Ann Rev Pharmacol 1969, 9:419-430.

6. Khanum F, Bawa AS, Singh B: Rhodiola rosea: A Versatile Adaptogen. Compr Rev Food Sci Food Saf 2005, 4(3):55-62.

7. Walker TB, Robergs RA: Does Rhodiola rosea possess ergogenic properties? Int J Sport Nutr Exerc Metab 2006, 16(3):305-315.

8. Brown RP, Gerbarg PL, Ramazanov Z: Rhodiola rosea: a phytomedicinal overview. HerbalGram 2002, 56:40-52.

9. Morgan M, Bone K: Rhodiola: The Arctic Adaptogen. Townsend Letter for Doctors and Patients. 2005, 262:26-28.

10. Kucinskaite A, Poblocka-Olech L, Krauze-Baranowska M, Sznitowska M, Savickas A, Briedis V: Evaluation of biologically active compounds in roots and rhizomes of Rhodiola rosea L. cultivated in Lithuania. Medicina (Kaunas) 2007, 43(6):487-494

11. Wiedenfeld H, Dumaa M, Malinowski M, Furmanowa M, Narantuya S: Phytochemical and analytical studies of extracts from Rhodiola rosea and Rhodiola quadrifida. Pharmazie 2007, 62(4):308-311. 
12. Mattioli L, Perfumi M: Effects of a Rhodiola rosea L. extract on acquisition and expression of morphine tolerance and dependence in mice. J Psychopharmacol 2011, 25(3):411-420.

13. De Merlier K, McGregor N: Chronic fatigue syndrome guidelines. J Chron Fatigue Syndr 2003, 11:1-5.

14. Sharpe M: Chronic fatigue syndrome: Neurological, mental or both. J Psychosom Res 2011, 70(6):498-499.

15. Sharpe MC, Archard LC, Banatvala JE, Borysiewicz LK, Clare AW, David A, Edwardss RH, Hawton KE, Lambert HP, Lane RJ: A report- chronic fatigue syndrome: guidelines for research. J R Soc Med 1991, 84(2):118-121.

16. Higgins JPT, Green S: Cochrane Handbook for Systematic Reviews of Interventions Version 5.0.0 [updated February 2008]. The Cochrane Collaboration, 2008. Available from www.cochrane-handbook.org.

17. Abidov M, Grachev S, Seifulla RD, Ziegenfuss TN: Extract of Rhodiola rosea radix reduces the level of $\mathrm{C}$-reactive protein and creatinine kinase in the blood. BE Biol Med 2004, 138(1):63-64.

18. Colson SN, Wyatt FB, Johnston DL, Autrey LD, FitzGerald YL, Earnest CP: Cordyceps sinensis- and Rhodiola rosea-based supplementation in male cyclists and its effect on muscle tissue oxygen saturation. J Strength Cond Res 2005, 19(2):358-363.

19. Darbinyan V, Kteyan A, Panossian A, Gabrielian E, Wikman G, Wagner H: Rhodiola rosea in stress induced fatigue-a double blind cross-over study of a standardized extract SHR- 5 with a repeated low-dose regimen on the mental performance of healthy physicians during night duty. Phytomedicine 2000, 7(5):365-371.

20. De Bock K, Eijnde BO, Ramaekers M, Hespel P: Acute Rhodiola rosea intake can improve endurance exercise performance. Int I Sport Nutr Exerc Metab 2004, 14(3):298-307.

21. Earnest CP, Morss GM, Wyatt F, Jordan AN, Colson S, Church TS, et al: Effects of a commercial herbal-based formula on exercise performance in cyclists. Med Sci Sports Exerc 2004, 36(3):504-509.

22. Olsson EM, von Scheele B, Panossian AG: A randomised, double-blind, placebo-controlled, parallel-group study of the standardised extract shr- 5 of the roots of Rhodiola rosea in the treatment of subjects with stress-related fatigue. Planta Med 2009, 75(2):105-112.

23. Shevtsov VA, Zholus BI, Shervarly VI, Vol'skij VB, Korovin YP, Khristich MP, et al: A randomized trial of two different doses of a SHR-5 Rhodiola rosea extract versus placebo and control of capacity for mental work phytomedicine. Phytomedicine 2003, 10(2-3):95-105.

24. Spasov AA, Wikman GK, Mandrikov VB, Mironova IA, Neumoin W: A double-blind, placebo-controlled pilot study of the stimulating and adaptogenic effect of Rhodiola rosea SHR-5 extract on the fatigue of students caused by stress during an examination period with a repeated low-dose regimen. Phytomedicine 2000, 7(2):85-89.

25. Walker TB, Altobelli SA, Caprihan A, Robergs RA: Failure of Rhodiola rosea to alter skeletal muscle phosphate kinetics in trained men. Metab Clin Exp 2007, 56(8):1111-1117.

26. Spasov AA, Mandrikov VB, Mironova IA: The effect of the preparation rodakson on the psychophysiological and physical adaptation of students to an academic load. Eksperimentalnaia i Klinicheskaia Farmakologiia 2000, 63(1):76-78.

27. Mattioli L, Perfumi M: Rhodiola rosea L. extract reduces stress- and CRF-induced anorexia in rats. J Psychopharmacol 2007, 21(7):742-750

28. Gluud LL: Bias in clinical intervention research. Am J Epidemiol 2006, 163(6):493.

29. Montori VM, Guyatt GH: Intention-to-treat principle. Can Med Assoc J 2001, 165(10):1339.

30. Luscher M: The Luscher Color Test. New York: Pocket Books; 1971.

31. Schulz K, Altman D, Moher D: CONSORT 2010 Statement: updated guidelines for reporting parallel group randomised trials. BMC Med 2010 $8(1): 18$.

32. Williamson EM: Interactions between herbal and conventional medicines. Expert Opin Drug Saf 2005, 4(2):355-378.

33. Fugh-Berman A, Ernst E: Herb-drug interactions: Review and assessment of report reliability. Br J Clin Pharmacol 2001, 52(5):587-595.

\section{Submit your next manuscript to BioMed Central and take full advantage of:}

- Convenient online submission

- Thorough peer review

- No space constraints or color figure charges

- Immediate publication on acceptance

- Inclusion in PubMed, CAS, Scopus and Google Scholar

- Research which is freely available for redistribution 